Nervenarzt 2020 • 91:1025-1031 https://doi.org/10.1007/s00115-020-00986-x Online publiziert: 8 . September 2020

(c) Springer Medizin Verlag GmbH, ein Teil von Springer Nature 2020

\section{Hintergrund}

Marginalisierungsprozesse bis hin zur Wohnungslosigkeit sind Ausdruck sozialer Exklusions- und Abstiegsphänomene, die das Gesundheitssystem in Verbindung mit anderen Teilhabeleistungen und Institutionen des Hilfs- und Versorgungssystems vor besondere Herausforderungen stellen. Verschiedene politische Entwicklungen der letzten Jahre, welche mit der zunehmenden Wohnungslosigkeit verbunden sind, wie beispielsweise Urbanisierung, Gentrifizierung und internationale Fluchtbewegungen, stellen damit Bestrebungen nach „Inklusion“ und „gesellschaftlichen Zusammenhalt" auf den Prüfstand. Die aktuelle COVID-19-Pandemie könnte bereits bestehende Momente der Benachteiligung verschärfen. Umfrageergebnisse der Bundesarbeitsgemeinschaft Wohnungslosenhilfe unter Einrichtungen der Wohnungslosenhilfe während der COVID-19-Pandemie weisen auf erhebliche Schwierigkeiten in der Steuerung bzw. der Aufrechterhaltung von Hilfsmaßnahmen für wohnungslose Menschen hin [7].

In der Vergangenheit waren Menschen in Wohnungslosigkeit wiederholt politischer Verfolgung ausgesetzt, was leider auch durch die Beschäftigung mit dem Phänomen Wohnungslosigkeit im psychiatrischen Kontext gefördert wurde $[25,49,60]$. Der Psychiater Kurt Schneider beschrieb beispielsweise 1934, dass

Die Autoren S. Schreiter und S. Gutwinski teilen sich die Erstautorenschaft.

\author{
S. Schreiter ${ }^{1} \cdot$ S. Gutwinski ${ }^{1,2} \cdot$ W. Rössler ${ }^{1,3}$ \\ ${ }^{1}$ Department of Psychiatry and Psychotherapy, Charité - Universitätsmedizin Berlin, corporate member of \\ Freie Universität Berlin, Humboldt-Universität zu Berlin, and Berlin Institute of Health, Berlin, \\ Deutschland \\ ${ }^{2}$ Psychiatrische Universitätsklinik der Charité im St. Hedwig Krankenhaus, Berlin, Deutschland \\ ${ }^{3}$ Klinik für Psychiatrie und Psychotherapie, Psychiatrische Universitätsklinik, Universität Zürich, Zürich, \\ Schweiz
}

\title{
Wohnungslosigkeit und seelische Erkrankungen
}

wohnungslose Menschen durch eine innere Unruhe sowie „eine triebhafte Sucht nach Veränderung und etwas Neuem“ gekennzeichnet seien und bezeichnete sie als „unstete Psychopathen“ [25, 49]. Solche als wissenschaftlich deklarierten Betrachtungen und deren Verbreitung im Gesamtkontext der historischen Entwicklung ebneten den Weg zur nationalsozialistischen Verfolgung von als „asozial“ bezeichneten Menschen, die in den 1930er- und 1940er-Jahren auf der Straße lebten $[23,25]$. Doch auch in der Wohnungslosenforschung der Nachkriegszeit findet sich weiterhin die Sichtweise von Obdachlosigkeit als Charakterstörung.

\section{》) Nur wenigen Personen gelingt es, die soziale Notlage zu beenden}

Durch eine steigende Arbeitslosigkeit sowie einer Zunahme der Mietpreise in den 1970er-Jahren und schließlich auch infolge der Wiedervereinigung stiegen die Zahlen wohnungsloser Menschen in Deutschland an. Wohnungslosigkeit wurde dabei zunehmend als eine Form neuer Armut, als ein Problem sozialer Ausgrenzungsprozesse verstanden. Durch einen in den vergangenen Jahrzehnten erneuten Anstieg der Wohnungslosenzahlen sowie eine zunehmende Verschiebung von Personen mit schweren psychischen Erkrankungen in den Versorgungsbereich der Wohnungslosenhilfe hat auch in den letzten Jahren erneut ein vermehrtes Forschungsinter- esse auf diesem Gebiet eingesetzt [10, 28, 48, 51, 52]. Trotz der Bemühungen der Gemeindepsychiatrie eine Versorgung im Lebensraum der Betroffenen zu ermöglichen, scheinen die Angebote nur einen Teil der Betroffenen zu entsprechen bzw. zu erreichen $[33,57,59]$. Trotz eines langjährig etablierten Versorgungssystems im Bereich der Wohnungslosenhilfe gelingt es nur wenigen Personen, diese soziale Notlage zu beenden. Ein erneutes Umdenken hinsichtlich neuer Lösungen zur Realisierung des Anspruchs auf gesellschaftliche Teilhabe auch für Personen in sozialen Notlagen unter der Einbeziehung der Betroffenenperspektive scheint notwendig.

Bisher liegen, abgesehen von einer ersten Berliner Zählung im Rahmen der „Nacht der Solidarität“, in der 1976 obdachlose Personen erfasst wurden [56], keine offiziellen Zahlen über wohnungslose Menschen in Deutschland vor $[8,56]$. Eine Schätzung der Bundesarbeitsgemeinschaft Wohnungslosenhilfe geht für 2018 von 678.000 Personen in Deutschland aus, einschließlich 441.000 anerkannten geflüchteten Menschen [8]. Demnach leben 41.000 der wohnungslosen Personen ohne jede Unterkunft auf der Straße [8]. Insbesondere der zunehmende Anteil auf der Straße lebender Familien wird dabei als alarmierend angemerkt. Der Anteil an Frauen lag bei geschätzten 59.000 (27\%); der Anteil der Kinder und Jugendlichen umfasste schätzungsweise 19.000 (8\%) Personen [8]. In einer Erhebung aus Österreich wurden 201721.567 wohnungslose Personen berichtet, von denen 13.900 als 


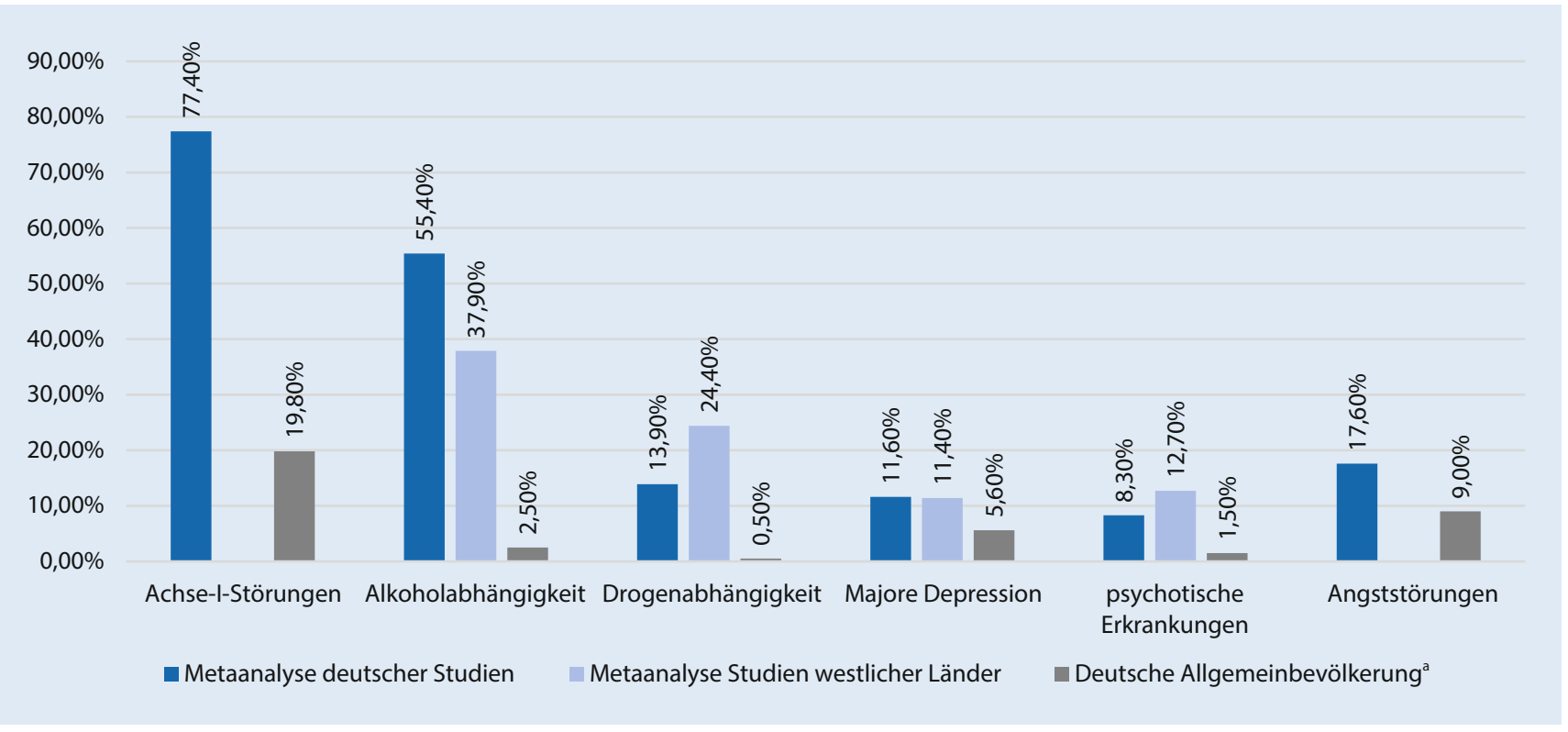

Abb. 1 ॥ Punktprävalenzen psychischer Erkrankungen unter wohnungslosen Menschen in Deutschland und in westlichen Ländern sowie der deutschen Allgemeinbevölkerung. Einmonatsprävalenz für Abhängigkeit und schädlichen Gebrauch zusammengefasst; Einmonatsprävalenz für jegliche unipolare Depression; Screening für Schizophrenie und andere psychotische Störungen ohne weitere Differenzialdiagnostik, umfasst ebenfalls psychotische Symptome bei affektiven Erkrankungen. (Quelle [21, 32, 51])

registrierte obdachlose Personen und 8700 Personen in Einrichtungen für wohnungslose Menschen in die Statistik Eingang fanden $[10,11]$. Zahlen für die gesamte Schweiz liegen unserer Kenntnis nach nicht vor, abgesehen von einer Erhebung aus Basel aus dem Jahr 2018 nach der 50 Personen auf der Straße, 50 Personen in Notunterkünften und weitere 200 Personen in Wohnungen der Sozialhilfe leben [12]. Insgesamt erscheinen für alle drei Länder strukturierte, fortlaufende Erhebungen sinnvoll, um eine zielgerichtete Erfassung von Hilfebedarfen zu ermöglichen. Dabei sollten Planung und Umsetzung derartiger Erhebungen aufgrund der durch die Zählung möglichen Stigmatisierung eng mit Vertretungen Betroffener abgestimmt werden (siehe auch Stellungnahme der Selbstvertretung wohnungsloser Menschen e. V. [54]).

Moderne Forschungskonzepte sind zuletzt zwar zunehmend ressourcenorientiert [48], trotzdem mangelt es häufig an der Einbeziehung von Betroffenen bei der Entwicklung innovativer Lösungen und Strategien. Eine erste Schwierigkeit liegt hierbei bereits in der Operationalisierung und der Entwicklung eines einheitlichen Verständnisses für den Begriff der Wohnungslosigkeit. Während der
Zählung in Berlin bezog man (orientiert an ähnlichen internationalen Zählungen) beispielsweise ausschließlich obdachlose Personen, die direkt auf der Straße lebten oder Angebote der Kältehilfe in Anspruch nahmen, mit ein und erfasste eine Zahl von 1976 Personen, die damit nur eine Teilgruppe der Betroffenen darstellen [56]. Andere Definitionen berücksichtigen darüber hinaus Personen, die z. B. in Obdachlosenwohnheimen, Frauenhäusern, Flüchtlingsunterkünften oder vorrübergehend bei Bekannten leben [20].

Insgesamt stellt das Problem der Wohnungslosigkeit eine multikausale Verkettung diverser Momente der Benachteiligung dar, die vermutlich nur in einer inter- und transdisziplinären Bearbeitung zugänglich ist und eine Einbeziehung von Tätigen im Feld, Betroffenen, Politik und wissenschaftlichen Akteuren der Medizin, Sozialwissenschaften, Stadtentwicklung etc. erforderlich macht. Im Folgenden sollen hierbei auf individuelle Aspekte sowie auch institutionelle Barrieren eingegangen werden.

\section{Wohnungslosigkeit und psychische Erkrankungen}

In welchem Maße sich die Entwicklung psychischer Krisen oder Störungen und soziale Prozesse bei wohnungslosen Menschen gegenseitig beeinflussen, bleibt bisher weitestgehend ungeklärt. Dass jedoch seelische Erkrankungen unter Menschen in der Extremsituation Wohnungslosigkeit gehäuft im Vergleich $\mathrm{zu}$ Erhebungen in der Allgemeinbevölkerung $\mathrm{zu}$ beobachten sind, zeigen zahlreiche nationale wie internationale Studien: Eine Metaanalyse der Universität Oxford zeigte hohe Punktprävalenzen seelischer Erkrankungen in westlichen Ländern [21]. Wobei sich in erster Linie Suchterkrankungen fanden (gepoolte Prävalenz der Alkoholabhängigkeit $37,9 \%$, gepoolte Prävalenz anderer Substanzabhängigkeiten 24,4\%), gefolgt von Persönlichkeitsstörungen (23,1\%), psychotischen Störungen (12,7\%) und Depressionen (11,4\%; [21]). Eine Metaanalyse deutscher Studien umfasste 1220 Personen in 11 Studien und ergab eine Einmonatsprävalenz psychischer Erkrankungen von $77,4 \%$ unter wohnungslosen Menschen [51]. Dem gegenüber stehen Zahlen der Allge- 
meinbevölkerung, die in der Regel ausschließlich Personen in einem geregelten Wohnverhältnis umfassen, mit einer Einmonatsprävalenz seelischer Störungen von 19,8\% (DEGS1 [Studie zur Gesundheit Erwachsener in Deutschland]; [32]; für eine zusammenfassende Darstellung siehe - Abb. 1).

Hohe Zahlen finden sind auch in Studien aus Österreich und der Schweiz [1, 12]. Eine Studie aus Salzburg erbrachte unter wohnungslosen Jugendlichen die Prävalenz seelischer Erkrankungen von $80 \%$ (an erster Stelle Suchterkrankungen mit $65 \%$, dann folgen affektive Erkrankungen mit 42,5\%, Angststörungen mit 17,5\% und Essstörungen mit 17,5\%; [1]). In Zürich wurden Prävalenzen seelischer Störungen bei wohnungslosen Personen sogar von über $96 \%$ berichtet; ebenfalls an erster Stelle Suchterkrankungen, 60,9\% Drogenabhängigkeit und 41,5\% Alkoholabhängigkeit, wobei die Prävalenzen laut Autoren etwas zu hoch liegen, da die Diagnostik nur mittels eines Kurzinterviews erfolgte [12].

Hervorzuheben ist, dass in der deutschen Metaanalyse die Raten psychotischer Erkrankungen und depressiver Störungen annähernd gleich hoch ausfallen, was in Kontrast zu anderen Gruppen in besonderen Lebenslagen wie geflüchtete Menschen oder Menschen in Haft für einen besonders hohen Anteil psychotischer Störungen unter Menschen in Wohnungslosigkeit spricht [21]. Diese Zahlen machen deutlich, dass eine Vielzahl der Personen auf der Straße unter Erkrankungen leiden, die zusätzlicher Unterstützung bedürfen, um den bestehenden Teilhabeanspruch zu ermöglichen [51]. Das hierfür gewachsene Versorgungssystem scheint jedoch nur unzureichend in der Lage zu sein, Schnittstellen zwischen Gesundheitsversorgungssystem und anderen Institutionen $\mathrm{zu}$ überwinden und Personen in Wohnungslosigkeit in ihrer Lebensrealität ausreichend zu erreichen und zu unterstützen. Sowohl stationäre Behandlungen sowie nur unzureichend aufsuchend organisierte ambulante Behandlungen werden den Bedarfen und Lebenslagen der Betroffenen häufig nicht gerecht, was sich in einer intensiven Nutzung des Notfall- und Akutversor-

Nervenarzt 2020 · 91:1025-1031 https://doi.org/10.1007/s00115-020-00986-x

(c) Springer Medizin Verlag GmbH, ein Teil von Springer Nature 2020

\section{S. Schreiter · S. Gutwinski · W. Rössler \\ Wohnungslosigkeit und seelische Erkrankungen}

\section{Zusammenfassung}

Wohnungslosigkeit ist Ausdruck ausgeprägter sozialer Exklusionsphänomene und trifft besonders häufig Menschen mit seelischen Erkrankungen. Hierbei gehen die Erkrankungen meist der Wohnungslosigkeit vorweg, wobei einige seelische Störungen erst infolge der Wohnungslosigkeit auftreten. International wurde eine Vielzahl therapeutischer Unterstützungsformen für wohnungslose Menschen mit seelischen Erkrankungen evaluiert mit vorwiegend aufsuchenden Behandlungsstrategien. Dies wird häufig kombiniert mit niedrigschwelliger Zurverfügungstellung von Wohnraum.
Hierbei zeigen sich gute Effekte auf die Zeit des Verbleibs in Wohnungen und auf die Verbesserung psychiatrischer Symptome, allerdings keine ausreichenden Effekte auf Substanzgebrauchsstörungen. Möglichkeiten zukünftiger Projekte liegen am ehesten im Einbezug ehemals Betroffener und in der Verwendung digitaler Medien.

\section{Schlüsselwörter}

Wohnungsnot · Armut · Substanzabhängigkeit . Aufsuchende Unterstützung . Krisenerfahrung

\section{Homelessness and mental illnesses}

\section{Abstract}

Homelessness is an expression of marked social exclusion phenomena and often particularly affects people with mental disorders. Mental disorders often precede the onset of homelessness but can also be a result of homelessness. Different forms of therapeutic and social support interventions have been evaluated in various countries, predominantly with an outreach treatment approach. These interventions were often combined with low threshold availability of housing programs. These showed positive effects on housing stability and reduction of psychiatric symptoms but not in reduction of substance use disorders. Peer support strategies and the use of digital media are possible options for future therapeutic strategies.

Keywords

Housing shortage $\cdot$ Poverty $\cdot$ Substancerelated addiction - Outreach support · Crisis experience gungssystems zeigt [30]. Aufgrund der weiten Verbreitung von Alkohol- und Substanzgebrauch liegt zudem ein besonderer Fokus auf dem Suchthilfebereich [27]. Hohe Zahlen von Abhängigkeitserkrankungen unter wohnungslosen Menschen - in Deutschland maßgeblich die Alkoholabhängigkeit entsprechend der einfachen Verfügbarkeit - sind dabei vermutlich Ausdruck einer CopingStrategie in marginalisierten Lebenswirklichkeiten [37].

Für das Versorgungssystem werden dabei relevante Fragen aufgeworfen: Wie können insbesondere unter Berücksichtigung der Überwindung von Schnittstellen Menschen mit Substanzgebrauchsstörungen besser in ihrem Recht auf gesellschaftliche Teilhabe unterstützt und somit auch vor Prozessen der Marginali- sierung geschützt werden und wie können Angebote des Suchthilfesystems auch die komplexen Bedarfe von Menschen in Wohnungslosigkeit besser adressieren [27, 28]?

Auch eine Fortsetzung der Diskussion um die Abstinenzorientiertheit des Suchthilfesystems wäre hierfür notwendig, da diese nicht immer mit der Lebenswirklichkeit der Betroffenen in Einklang steht [27].

\section{》) Der Erkrankungsbeginn liegt in der Regel vor dem Beginn der Wohnungslosigkeit}

Hinsichtlich des Zeitpunkts des Einsetzens psychischer Symptomatik im Verhältnis zum Zeitpunkt des Wohnungs- 
verlustes zeigen Studien, dass in der Regel der Erkrankungsbeginn vor dem Beginn der Wohnungslosigkeit liegt [43]. In der Seewolf-Studie aus dem Großraum München waren $85 \%$ der Untersuchten vor oder während ihres Wohnungsverlustes in stationär-psychiatrischer Behandlung befindlich [10]. In einer Studie aus dem US-amerikanischen Raum lag bei männlichen wohnungslosen Personen der Erkrankungsbeginn einer Schizophrenie in $75 \%$ und bei einer bipolaren affektiven Störung in $76 \%$ der Fälle vor Beginn der Wohnungslosigkeit [43]. Bei Frauen fielen diese Werte für einige Erkrankungsbilder deutlich höher aus: Der Erkrankungsbeginn lag bei Schizophrenie $\mathrm{zu} 100 \%$ und bei bipolaren affektiven Störungen zu $80 \%$ vor Beginn der Wohnungslosigkeit [43]. Ähnlich fielen die Ergebnisse für die Alkoholabhängigkeit aus (Männer 87,7\% und Frauen 83,7\%; [43]).

Dabei nahmen laut der Berliner Wohin-Studie Menschen in Wohnungslosigkeit, die sich in (teil-)stationärer psychiatrischer Behandlung befanden, durchschnittlich früher im Leben ambulante und stationäre Behandlungen wahr als Patient*Innen in geregelten Wohnverhältnissen (Alter der ersten stationärpsychiatrischen Behandlung 23,9 Jahre vs. 34,5 Jahre; [52]). Dieser Befund zeigt sich erkrankungsübergreifend und besonders für wohnungslose Menschen mit Suchterkrankungen [52].

Da ein früher Krankheitsbeginn bei vielen seelischen Erkrankungen mit wichtigen Schwellensituationen zusammenfällt, erscheint die damit assoziierte häufigere Wohnungslosigkeit als Ausdruck der verbundenen Schwierigkeiten der sozialen Entwicklung [27]. Der Beginn dieser sozialen Prozesse liegt vermutlich häufig bereits in einem vorzeitigen Abbruch der Schule: In der Berliner Wohin-Studie berichteten $28,3 \%$ der psychiatrisch behandelte wohnungslose Patient ${ }^{\star}$ Innen, keinen Schulabschluss zu haben, gegenüber 9,7\% der Patient ${ }^{\star}$ Innen in geregelten Wohnverhältnissen [52]. Niedrigschwellige Hilfsangebote bereits für junge Menschen in seelischen Krisen zu etablieren, beispielsweise unter der Einbeziehung altersentsprechender webbasierter Lö- sungen sowie durch eine enge Zusammenarbeit im Bereich der Erwachsenen-, Kinder- und Jugendpsychiatrie und Jugendhilfe, scheint demnach sinnvoll.

Gleichermaßen ist davon auszugehen, dass die Wohnungslosigkeit als Stressor das Auftreten seelischer Störungen begünstigt: In der Studie von North et al. sind über $30 \%$ der generalisierten Angsterkrankungen und depressiven Störungen nach Beginn der Wohnungslosigkeit aufgetreten [43].

\section{Belastende Lebensereignisse und andere Barrieren}

Die Bedeutung belastender Lebensereignisse im Kindesalter sowie im späteren Erwachsenenalter unter Menschen, die in die Notlage der Wohnungslosigkeit geraten sind, wurde über viele Jahre nicht ausreichend berücksichtig. Studien aus Kanada zeigen, dass beispielsweise etwa $50 \%$ der wohnungslosen Personen in kanadischen Städten Erfahrungen mit Pflegefamilien (,foster care“) gemacht hatten [38], was nahe legt, dass viele dieser Personen aus einem Umfeld kamen, in dem es zu keiner ausreichenden Versorgung der Kinder gekommen war. In einer kanadischen Studie, die 500 wohnungslose Personen untersuchte, berichteten insgesamt 63,6\% der Befragten über Erfahrungen frühkindlicher Traumatisierungen, 57,7\% über körperlichen Missbrauch, 62,8 \% über körperliche Vernachlässigung, 45,8\% über sexuellen Missbrauch, $62,4 \%$ über emotionalen Missbrauch und $55,2 \%$ über emotionale Vernachlässigung [55]. Vergleichbare Studienergebnisse aus Europa liegen bis auf eine Erhebung aus Großbritannien unserer Kenntnis nach nicht vor [58].

\section{》) Erfahrungen des sozialen Stigmas führen zu Mechanismen der Selbstbeschuldigung}

Zudem kommt es gehäuft bei Personen auf der Straße, die frühkindliche Traumaerfahrungen haben, gehäuft zu erneuten Diskriminierungs- und Gewalterlebnissen [18, 19]. Häufig erwarten Menschen in Wohnungslosigkeit basierend auf diskriminierenden Vorerfahrungen erneuter Stigmatisierung auch in Einrichtungen des Gesundheitssystem ausgesetzt $\mathrm{zu}$ sein und meiden dieses; erhebliches Misstrauen in die Behandlung kann daraus resultieren [2, 22, 35]. Dabei führen Erfahrungen des sozialen Stigmas "Wohnungslosigkeit" zu Mechanismen der Selbstbeschuldigung für die eigene Situation $[34,39]$ sowie zu Beeinträchtigungen des Selbstwertgefühls [13]. Zudem kann sich aus den erlebten Erfahrungen ein Gefühl ständiger Angst vor Übergriffen ergeben [42].

Derartige internalisierte kognitive wie emotionale Barrieren treffen hierbei auf externe Barrieren des Versorgungssystems und Alltagslebens und werden erschwert durch individuelle Risikofaktoren wie frühkindliche Traumatisierungen, Verlust des sozialen Netzes oder Vulnerabilität für eine psychische Erkrankungen und beeinflussen die Inanspruchnahme von Hilfen des Versorgungssystems negativ [45]. Externe Barrieren umfassen hierbei die direkte physische sowie zeitliche Erreichbarkeit, komplexe administrative Abläufe, Systeme der Terminvergabe, Wartelisten und andere administrative Regeln und fehlende angemessene Informationen eines häufig komplexen Hilfesystems [45]. Die Entwicklung geeigneter Versorgungsangebote, die die genannten Barrieren und komplexen Bedarfe berücksichtigen, werden benötigt; insbesondere auch unter stärkerer Berücksichtig möglicher Traumaerfahrungen und Erfahrungen von Stigmatisierungen [50].

\section{Ausblick - Strategien und Möglichkeiten}

Die Unterstützung wohnungsloser Menschen unterscheidet sich in vielen Fällen durch die Priorisierung sozialer existenzieller Probleme, den fehlenden festen Bezugsort, einen mitunter chaotischen Alltag und Armut von der Behandlung von Personen in einem festen Wohnumfeld [53].

\section{Aufsuchende Unterstützung}

Laut internationalen Studien haben sich aufsuchende Modelle der psychosozialen Unterstützung etabliert [6]. Dies umfasst 
in Deutschland unterschiedliche Modelle des „case managment"; dies sind derzeit vorwiegend nichtmedizinische Interventionen, wie ambulante Soziotherapie, rechtliche Betreuung und teilweise auch aufsuchende Arbeit sozialpsychiatrischer Dienste. Schwierigkeiten bestehen dabei häufig in den komplexen administrativen Abläufen; zudem unterscheiden sich angebotene Unterstützungsformen in Deutschland häufig durch ihre eingeschränkte zeitliche Verfügbarkeit entgegen der Forderung nach einer intensiven, bedarfsgerechten Unterstützung in Form eines multiprofessionellen Teams rund um die Uhr [6].

International liegt eine Vielzahl von Publikationen vor, die die Wirksamkeit von Interventionen des ,intensive case management“ (ICM) und des „assertive community treatment" (ACT) in der Versorgung Wohnungsloser belegen $[6,28$, 48]. Beide Verfahren zeichnen sich durch einen höheren Personalschlüssel gegenüber klassischen Case-management-Angeboten aus - das ACT beinhaltet zudem eine multiprofessionelle 12-h- bzw. 24-hBetreuung [14, 17]. Für das ACT konnte in mehreren Studien gezeigt werden, dass es gegenüber dem klassischen „case managment“ zu einem Rückgang der psychiatrischen Symptomatik kommt [15, $62,63]$. Nach stationären Behandlungen hat sich als Übergang in die ambulante Behandlung die „critical time intervention“ (CTI) bezüglich einer Verminderung der psychischen Symptomatik als wirksam erwiesen [17].

Eine überzeugende Wirksamkeit der genannten Verfahren bei wohnungslosen Menschen mit Substanzgebrauchsstörungen im Hinblick auf die Reduktion des Substanzmittelkonsum konnte bisher nicht ausreichend belegt werden [28]. Auf „harm reduction“ ausgerichtete Versorgungsmodelle, die es in Deutschland teilweise gibt [41], auch hinsichtlich ihrer Wirksamkeit auf die Versorgung von Personen in Wohnungslosigkeit und Armut zu evaluieren, erscheint vielversprechend.

\section{„Housing first"}

„Housing first“ (HF) umfasst, dass Menschen bedingungslos Wohnraum zur
Verfügung gestellt bekommen; unabhängig hiervon sollen den Nutzern flexible Hilfen und Angebote der Unterstützung entsprechend der individuellen Bedarfe und Ressourcen zur Verfügung gestellt werden [44]. HF wirkte sich in mehreren Studien positiv auf soziale Parameter aus, wie die Dauer des Verbleibs in stabilen Wohnverhältnissen und eine verringerte Zahl von Kontakten mit dem Justizsystem [3, 4, 36, 46]. Bezogen auf eine psychische Symptomatik scheint sich die Wirksamkeit von HF laut longitudinaler Studien nur in Kombination mit ergänzenden Unterstützungen $\mathrm{zu}$ erweisen, wie die Verbindung von HF mit ACT [3, 4, 11, 26, 61]. Entscheidend scheint also nicht nur das zur Verfügung stellen von Wohnraum im Rahmen von HF-Modellen, was ebenfalls einen Ein- bezug von Akteuren von Politik und Wohnungsmarkt nötig macht, sondern auch die entsprechende Ausrichtung des Versorgungssystems, um angemessene Unterstützungsmöglichkeiten begleitend zu etablieren [44]. Erste Schritte hinsichtlich einer Entkopplung von Wohnen und sonstigen Unterstützungsleistungen hin zu Formen des „supported housing“ wie "housing first" sind bereits im Bundesteilhabegesetzt enthalten. Dies entspricht somit den Empfehlungen der aktuellen S3-Leitlinie Psychosoziale Therapien bei schweren psychischen Erkrankungen, ein selbstbestimmtes Wohnen in der Gemeinde und entsprechende an den individuellen Bedarfen und Präferenzen orientierte mobile Unterstützung zu ermöglichen [6].

\section{Hier steht eine Anzeige.}

\section{勿 Springer}




\section{Krisenerfahrungen und neue Kommunikationsformen}

Bereits Kim Hopper wies auf die Bedeutung überwundener und erlebter Krisenerfahrung hin und die damit verbundenen Ressourcen, ein Modell des Wegs des "recovery" zurück in die "community“ auch für andere Betroffene zur Verfügung zu stellen $[5,29]$. Das Einbeziehen erlebter Erfahrung in Prozesse der Versorgung und Forschung, wie es sich beispielsweise in Form der psychosozialen Beratung insbesondere von Menschen in Krisengebieten oder Fluchterfahrungen durch IPSO (International Psychosocial Organisation; [31]) etabliert hat, könnte mögliche Barrieren verringern. Vorteil des „peer support“ ist ein besonders schneller und tiefer Aufbau einer vertrauensvollen Beziehung [40]. „Peer support“ unterstützt auf Betroffenenseite hinsichtlich ihrer Selbstwirksamkeit [40]. Sie stellt eine Brücke in die Gesellschaft und ins Hilfssystem dar. Einzelne Studien konnten einen signifikanten positiven Effekt von „peer support“ auch bei wohnungslosen Erwachsenen und Jugendlichen auf Parameter wie Lebensqualität, Drogen- bzw. Alkoholkonsum und soziale Unterstützung zeigen [9], wobei dieses Feld kaum untersucht ist. Die Etablierung von „peer support“ an der Schnittstelle von psychischer und sozialer Krise wie der der Wohnungslosigkeit stellt jedoch eine besondere Herausforderung dar, da hierfür andere etablierte Hilfen ausreichend gut abgestimmt sein müssen [16, 29].

Gleichzeitig bieten sich in den sich veränderten Kommunikationsmustern und -wegen neue Möglichkeiten. Wie können beispielsweise Schnittstellenprobleme durch digitale Lösungen verbessert werden oder Beratungsangebote auch online zur Verfügung gestellt werden? Entgegen Befürchtungen verwenden auch wohnungslose Menschen Smartphones, Internetzugang und Plattformen sozialer Medien: Studien zeigten, dass mehr als $80 \%$ wohnungsloser Jugendlicher mehr als einmal pro Woche online gehen und ungefähr ein Viertel nutzt das Internet für mehr als eine Stunde täglich; über $80 \%$ nutzen mindestens einmal in der Woche Plattformen sozialer Medien
[47]. Viele Wohnungslose haben eine Facebook-Adresse, aber kein physisches Zuhause. Erste Studienergebnisse aus dem amerikanischen Raum konnten hierbei positive Befunde hinsichtlich der Akzeptanz und Nutzung partizipativ entwickelter App-Anwendungen für Jugendliche und junge Erwachsene in Wohnungslosigkeit belegen [24].

\section{Schlussfolgerung}

Wohnungslosigkeit stellt ein komplexes gesellschaftliches Phänomen der sozialen Exklusion dar. Sowohl im Hinblick auf den Einfluss psychischer Erkrankungen als auch in der Gestaltung von Hilfsangeboten seitens der Psychiatrie für Personen in sozialen Notlagen stellt Wohnungslosigkeit eine besondere Herausforderung dar. Dabei ist zu bedenken, dass sowohl der Beginn psychischer Krisen als auch der Zeitpunkt der Inanspruchnahme des psychiatrischen Versorgungssystems in der Regel vor dem Verlust der Wohnung liegen. Substanzgebrauch, Erfahrungen frühkindlicher Traumatisierungen und internalisierte Barrieren im Rahmen von Erfahrungen institutioneller und gesellschaftlicher Ausgrenzung gilt es, stärker $\mathrm{zu}$ berücksichtigen. Bisherige etablierte Unterstützungsangebote müssen hinsichtlich eines internationalen und evidenzbasierten Standards und möglicher externer Barrieren überprüft werden. Mögliche Lösungen können hierbei in der Nutzung webbasierter Angebote und dem stärkeren Einbezug der Betroffenenperspektive und von „peer supports“ liegen.

\section{Fazit für die Praxis}

- Wohnungslose Menschen sind überproportional häufig von seelischen Erkrankungen betroffen.

- Substanzgebrauchsstörungen, affektive Störungen und Psychosen sind die häufigsten Diagnosen unter wohnungslosen Menschen mit seelischen Erkrankungen.

- Traumatisierungen im Kindesalter werden als Faktor bisher in der Entstehung von Wohnungslosigkeit wenig berücksichtigt.
- Vielversprechende Strategien liegen in aufsuchender Behandlung und der Peer-Arbeit sowie niedrigschwelliger Zurverfügungsstellung von Wohnraum.

\begin{tabular}{l} 
Korrespondenzadresse \\
$\begin{array}{l}\text { Dr. S. Schreiter } \\
\text { Department of Psychiatry } \\
\text { and Psychotherapy, Charité - } \\
\text { Universitätsmedizin Berlin, } \\
\text { corporate member of Freie } \\
\text { Universität Berlin, Humboldt- } \\
\text { Universität zu Berlin, and } \\
\text { Berlin Institute of Health } \\
\text { Charitéplatz 1, 10117 Berlin, } \\
\text { Deutschland } \\
\text { Stefanie.Schreiter@charite.de }\end{array}$ \\
\hline
\end{tabular}

\section{Einhaltung ethischer Richtlinien}

Interessenkonflikt. S. Schreiter, S. Gutwinski und W. Rössler geben an, dass kein Interessenkonflikt besteht.

Für diesen Beitrag wurden von den Autoren keine Studien an Menschen oder Tieren durchgeführt. Für die aufgeführten Studien gelten die jeweils dort angegebenen ethischen Richtlinien.

\section{Literatur}

1. Aichhorn W, Santeler S, Stelzig-Scholer R et al (2008) Prevalence of psychiatric disorders among homeless adolescents. Neuropsychiatrie 22:180-188

2. Atterbury J (2010) Fair access for all? Gypsies and travellers in Sussex, GP surgeries and barriers to primary healthcare. http://www.gypsy-traveller org/pdfs/fair_access_health.pdf. Zugegriffen: 3 . März 2018

3. Aubry T, Goering P, Veldhuizen S et al (2016) A multiple-city RCT of housing first with assertive community treatment for homeless canadians with serious mental illness. Psychiatr Serv 67:275-281

4. Aubry T, Nelson G, Tsemberis S (2015) Housing first for people with severe mental illness who are homeless: a review of the research and findings from the at home-chez soi demonstration project. Canadian journal of psychiatry. Can J Psychiatry 60:467-474

5. Austin E, Ramakrishnan A, Hopper K (2014) Embodying recovery: a qualitative study of peer work in a consumer-run service setting. Community Ment Health J 50:879-885

6. DGPPN - Deutsche Gesellschaft für Psychiatrie und Psychotherapie, Psychosomatik und Nervenheilkunde (Hrsg) (2018) S3-Leitlinie Psychosoziale Therapien bei schweren psychischen Erkrankungen S3-Praxisleitlinien in Psychiatrie und Psychotherapie, 2. Aufl. https://www. awmf.org/uploads/tx_szleitlinien/038-020l_ S3_Psychosoziale_Therapien_bei_schweren psychischen_Erkrankungen_2019-07.pdf. Zugegriffen:01.06.2020 
7. Bag-W (2020) Ergebnisse der BAG W-Umfrage: CORONA und Wohnungslosigkeit (Aktualisiert am 23.04.2020). https://www.bagw.de/de/ neues 178.html. Zugegriffen:01.06.2020

8. Bag-W (2019)Zahl derWohnungslosen in Deutschland auf neuem Höchststand. https://www.bagw. de/de/themen/zahl_der_wohnungslosen/index. html.Zugegriffen:01.06.2020

9. Barker SL, Maguire N (2017) Experts by experience: peer support and its use with the homeless. Community Ment Health J53:598-612

10. Bäuml J, Brönner M, Baur B (2017) Die SEEWOLFStudie: Seelische Erkrankungsrate in den Einrichtungen der Wohnungslosenhilfe im Großraum München. Lambertus, Freiburg

11. Benston EA (2015) Housing programs for homeless individuals with mental illness: effects on housing and mental health outcomes. Psychiatr Serv 66:806-816

12. Briner D, Jager M, Kawohl W et al (2017) Mental disorder and self-rated health among homeless people in Zurich - first epidemiological data from Switzerland. Psychiat Prax 44:339-347

13. Campbell DJ, O'neill BG, Gibson $\mathrm{K}$ et al (2015) Primary healthcare needs and barriers to care among Calgary's homeless populations. BMC Fam Pract 16:139

14. Coldwell CM, Bender WS (2007) The effectiveness of assertive community treatment for homeless populations with severe mental illness: a metaanalysis. Am J Psychiatry 164:393-399

15. Cooper RL, Seiters J, Davidson DL et al (2010) Outcomes of integrated assertive community treatment for homeless consumers with cooccurring disorders. J Dual Diagn 6:152-170

16. Cubellis L (2018) Care wounds: precarious vulnerability and the potential of exposure. Cult Med Psychiatry 42:628-646

17. De Vet R, Van Luijtelaar MJ, Brilleslijper-Kater SN et al (2013) Effectiveness of case management for homeless persons: a systematic review. Am J Public Health 103:e13-26

18. Edalati H, Krausz M, Schutz CG (2016) Childhood maltreatment and revictimization in a homeless population. J Interpers Violence 31:2492-2512

19. Edalati H, Nicholls TL (2019) Childhood maltreatment and the risk for criminal justice involvement and victimization among homeless individuals: a systematic review. Trauma Violence Abus 20:315-330

20. Europäische Kommission (2007) Messung der Obdachlosigkeit in Europa. http://ec.europa. $\mathrm{eu} /$ social/BlobServlet?docld=1998\&langld=de. Zugegriffen:27.Apr. 2020

21. Fazel S, Khosla V, Doll H et al (2008) The prevalence of mental disorders among the homeless in western countries: systematic review and metaregression analysis. PLoS Med 5:e225

22. FlickU, Rohnsch G (2007) Idealization and neglect: health concepts of homeless adolescents. J Health Psychol 12:737-749

23. Garcia C (1986) Karl Wilmanns and the vagabonds. Nervenarzt 57:227-232

24. Glover AC, Schueller SM, Winiarski DA et al (2019) Automated mobile phone-based mental health resource for homeless youth: pilot study assessing feasibility and acceptability. JMIR Ment Health 6:e15144

25. Greifenhagen A, Fichter M (1996) Psychiatric homelessness research. From "psychopathology of the vagrant" to "homeless mentally ill". Nervenarzt 67:905-910

26. Gühne U, Stein J, Weinmann S et al (2017) Housing interventions in severe mental ill- ness - international evidence from RCTs. Psychiat Prax 44:194-205

27. Gutwinski S, Schreiter S (2020) Mentally ill more affected by homelessness. Psychiat Prax 47:115-117

28. Hoell A, Salize HJ (2018) Die psychiatrische Versorgung von wohnungslosen Menschen mit psychischen Problemen. Spectr Psychiatr 2:8-12

29. Hopper K (2003) Reckoning with homelessness. Cornell University Press, Ithaca

30. Hwang SW, Chambers C, Chiu S et al (2013) A comprehensive assessment of health care utilization among homeless adults under a system of universal health insurance. Am J Public Health 103(Suppl):294-301

31. Ipso International Psychosocial Organisation. https://ipsocontext.org/de/. Zugegriffen: 01.06.2020

32. Jacobi F, Wittchen HU, Holting C et al (2004) Prevalence, co-morbidity and correlates of menta disorders in the general population: results from the German Health Interview and Examination Survey (GHS). Psychol Med 34:597-611

33. Jenderny S, Schreiter J, Steinhart I (2020) Psychiatric facilities in Germany-transparency and structures. Psychiatr Prax. https://doi.org/10. 1055/a-1126-1103

34. Kidd SA (2007) Youth homelessness and social stigma. J Youth Adolesc 36:291-299

35. Klop HT, Evenblij K, Gootjes JRG et al (2018) Care avoidance among homeless people and access to care: an interview study among spiritua caregivers, street pastors, homeless outreach workers and formerly homeless people. BMC Public Health 18:1095

36. Kozloff N, Adair CE, Palma Lazgare LI et al (2016) "Housing first" for homeless youth with mental illness. Pediatrics. https://doi.org/10.1542/peds. 2016-1514

37. Krausz M, Strehlau V, Schuetz CG (2016) Obdachlos, mittellos, hoffnungslos - Substanzkonsum, psychische Erkrankungen und Wohnungslosigkeit: ein Forschungsbericht aus den USA und Kanada. Suchttherapie 17:1-9

38. Krausz RM, Clarkson AF, Strehlau V et al (2013) Mental disorder, service use, and barriers to care among 500 homeless people in 3 different urban settings. Soc Psychiatry Psychiatr Epidemio 48:1235-1243

39. Lynn-Callo V (2000) Medicalizing homelessness: the production of self-blame and self-governing within homeless shelters. Med Anthropol Q 14:328-334

40. Mahlke C, Schulz G, Sielaff G et al (2019) Models of peer support in psychiatric care. Bundesgesundheitsblatt Gesundheitsforschung Gesundheitsschutz 62:214-221

41. Majic T, Gutwinski S (2020) Krisenintervention bei Menschen, die psychoaktive Substanzen konsumieren. In: Gahleitner S, Gutwinski S, Ortis-Mueller W (Hrsg) Praxis Krisenintervention. Kohlhammer, Stuttgart (In Press)

42. Mcneil R, Shannon K, Shaver L et al (2014) Negotiating place and gendered violence in Canada's largest open drug scene. Int J Drug Policy 25:608-615

43. North CS, Pollio DE, Smith EMetal (1998) Correlates of early onset and chronicity of homelessness in a large urban homeless population. J Nerv Ment Dis 186:393-400

44. O'campo P, Stergiopoulos V, Nir P et al (2016) How did a Housing First intervention improve health and social outcomes among homeless adults with mental illness in Toronto? Two-year outcomes from a randomised trial. BMJ Open 6:e10581
45. O'carroll A, Wainwright D (2019) Making sense of street chaos: an ethnographic exploration of homeless people's health service utilization. Int J Equity Health 18:113

46. Poremski D, Stergiopoulos V, Braithwaite E et a (2016) Effects of housing first on employment and income of homeless individuals: results of a randomized trial. Psychiatr Serv 67:603-609

47. Rice E, Monro W, Barman-Adhikari A et al (2010) Internet use, social networking, and HIV/AIDS risk for homeless adolescents. J Adolesc Health 47:610-613

48. Salize HJ, Arnold M, Uber E et al (2017) Improving mental health care in people at risk for getting homeless. Psychiat Prax 44:21-28

49. Schneider K (1934) Die psychopathischen Persönlichkeiten, 3. Aufl. Deuticke, Leipzig

50. Schomerus G, Stolzenburg S, Freitag S et al (2019) Stigma as a barrier to recognizing personal mental illness and seeking help: a prospective study among untreated persons with mental illness. Eur Arch Psychiatry Clin Neurosci 269:469-479

51. Schreiter S, Bermpohl F, Krausz M et al (2017) The prevalence of mental illness in homeless people in Germany. Dtsch Arztebl Int 114:665-672

52. Schreiter S, Heidrich S, Zulauf J et al (2019) Housing situation and healthcare for patients in a psychiatric centre in Berlin, Germany: a crosssectional patient survey. BMJ Open 9:e32576

53. Schreiter S, Rössler W, Heinz A et al (2020) Bank account ownership among people in psychiatric care in Berlin, Germany-A cross-sectional patien survey. Front Psychiatry. https://doi.org/10.3389/ fpsyt.2020.00508

54. Selbstvertretung Wohnungsloser Menschen e.V. (2020) Aufruf zur Kundgebung in Berlin. http:// www.wohnungslosentreffen.de/blog/208-202002-29-aufruf-zur-kundgebung-in-berlin.html. Zugegriffen:01.06.2020

55. Song MJ, Nikoo M, Choi F et al (2018) Childhood trauma and lifetime traumatic brain injury among individuals who are homeless. J Head Trauma Rehabil 33:185-190

56. Soziales SFlaU (2020) Erste Ergebnisse der Nacht der Solidarität. https://www.berlin.de/nacht-dersolidaritaet/ergebnisse/.Zugegriffen:01.06.2020

57. Steinhart I, Jenderny S, Schreiter J (2020) (Closed) psychiatric homes as part of the regional networks in Germany? Psychiatr Prax. https://doi.org/10. 1055/a-1126-1214

58. Sundin EC, Baguley T (2015) Prevalence of childhood abuse among people who are homeless in Western countries: a systematic review and meta-analysis. Soc Psychiatry Psychiatr Epidemiol 50:183-194

59. Wienberg G, Steinhart I (2020) The functional basic model for the psychiatric care of persons with severe mental illness - an update. Psychiat Prax 47:9-15

60. Wilmanns K (1906) Zur Psychopathologie des Landstreichers. Barth, Leipzig

61. Woodhall-Melnik J, Dunn JR (2016) A systematic review of outcomes associated with participation in Housing First programs. Hous Stud 31:287-304

62. Young MS, Barrett B, Engelhardt MA et al (2014) Six-month outcomes of an integrated assertive community treatment team serving adults with complex behavioral health and housing needs. Community Ment Health J 50:474-479

63. Young MS, Clark C, Moore K et al (2009) Comparing two service delivery models for homeless individuals with complex behavioral health needs: preliminary data from two SAMHSA treatment for homeless studies. J Dual Diagn 50:287-230 